\title{
Identification of Laminin Domains Involved in Branching Morphogenesis: Effects of Anti-Laminin Monoclonal Antibodies on Mouse Embryonic Lung Development
}

\author{
Lucia Schuger, ${ }^{*}$ Amy P. N. Skubitz, $†$ K. Sue O’Shea,, Jane F. Chang, ${ }^{*}$ and James Varani* \\ Departments of ${ }^{*}$ Pathology and $\$$ Anatomy, University of Michigan Medical School, Ann Arbor, Michigan 48109; and $\nmid$ Department \\ of Laboratory Medicine and Pathology, University of Minnesota Medical School, Minneapolis, Minnesota $55 / 55$
}

Accepted May 3, 1991

\begin{abstract}
We recently found that polyclonal antibodies to laminin, a basement membrane-related glycoprotein, inhibited murine lung morphogenesis when added to organ cultures of mouse embryonic lung. Using a series of monoclonal antilaminin antibodies with previously characterized subunit specificity (termed AL-1, AL-2, AL-3, AL-4, and AL-5), the deposition and functional involvement of different laminin domains in the developing lung were investigated. By immunohistochemistry the antibodies' reactivity was largely localized to the basement membrane, but was also present diffusely in the extracellular matrix throughout the mesenchyme. Organ cultures of lung cxplants from Day 12 embryos were cultured for 3 days in the presence of $50-100 \mu \mathrm{g} / \mathrm{ml}$ of each antibody or in the presence of the same concentration of immunoglobulins $\mathrm{G}$ and $\mathrm{M}$, laminin-neutralized antibody, or medium alone. Cultures were monitored by phase-contrast microscopy, light microscopy, and immunofluorescence. Although all antibodies penetrated the tissues in culture, only two of them inhibited branching activity. These two antibodies were AL-1, which binds on or near the cross region of laminin, and $\mathrm{AL}-5$, which binds to the lateral short arms at the globular end regions of the $\mathrm{B}$ chain of laminin. Inhibition of branching with these two antibodies was dose-dependent and statistically significant for the two concentrations used. AL-2, AL-3, AL-4, laminin-neutralized antibodies and control immunoglobulins did not alter lung morphogenesis. The two domains of laminin that promote lung branching morphogenesis have been reported by others to promote the attachment of a variety of cells and/or bind heparin. These domains of laminin may promote branching morphogenesis by facilitating cell attachment and, consequently, cell proliferation. 1991 Academic Press, Inc.
\end{abstract}

\section{INTRODUCTION}

Laminin is a multidomain glycoprotein localized mainly in basement membranes. As isolated from the Englebreth-Holm-Swarm (EHS) tumor and many other tissues, laminin is composed of three polypeptide chains, referred to as B1 $\left(M_{\mathrm{r}} 222,000\right), \mathrm{B} 2\left(M_{\mathrm{r}} 210,000\right)$, and $\mathrm{A}\left(M_{\mathrm{r}} 4,000,000\right)$. The chains are held together by disulfide bonds to form a cross-shaped molecule (Timpl et al., 1979). The complete amino acid sequences of all three chains of EHS laminin have been recently published (Sasaki et al., 1987; Sasaki and Yamada, 1987; Sasaki et al., 1988). Laminin has diverse effects on a variety of cells, including stimulation of adhesion, migration, growth, and differentiation (Martin and Timpl, 1987; Kleinman et al., 1987a). Studies with proteolytic fragments, domain-specific antibodies, and synthetic peptides have identified different regions of laminin with biological activity. Two of the major cell binding regions are localized at the cross intersection (Terranova et al., 1983; Skubitz et al., 1987; Graf et al., 1987; Charonis et al., 1988; Kleinman et al., 1989) and at the terminal end of the long arm (Aumailley et al., 1987; Goodman et al., 1987; Tashiro et al., 1989).
Laminin is already present in early preimplantation embryos (Cooper and MacQueen, 1983) and thereafter is largely restricted to basement membranes of a variety of tissues during all stages of development (Wu et al., 1983). Recent studies of kidney organogenesis have shown that the different laminin chains are not coordinately expressed during embryogenesis, suggesting that molecular isoforms might also exist (Ekblom et al., 1990; Kleinman et al., 1987b; Laurie et al., 1989). Relying, in part, on the existence of these spatial and temporal distributions of the molecule and their coincidence with key developmental events, laminin has been postulated to be instrumental for organ development (Ekblom et al., 1980, 1986, 1990; Martin and Timpl, 1987).

In a recent study, we established that laminin plays an important role in the morphogenesis of embryonic mouse lung. Using polyclonal antibodies to laminin, we demonstrated that the antibodies were able to penetrate lung tissue in organ culture, localize at the basement membrane, and interfere with branch formation. Antibodies to two other components of the extracellular matrix, thrombospondin and entactin, failed to alter the normal pattern of development (Schuger et al., 1990; unpublished). These findings are extended in the current 

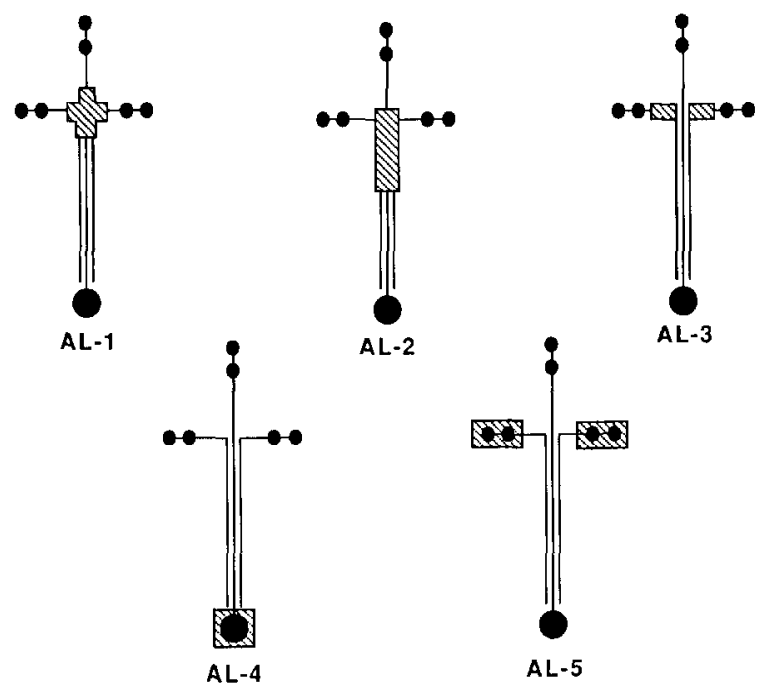

Fig. 1. Diagrammatic models of the monoclonal antibody binding sites on laminin Anti-laminin monoclonal antibodies AL-1, Al-2, Al-3, $\mathrm{AL}-4$, and $\mathrm{AL}-5$ were incubated with laminin and analyzed by electron microscopy after rotary shadowing. (Skubitz et al., 1987, 1988).

investigation using a series of monoclonal antibodies to distinct domains of laminin. We demonstrate that the inhibition observed is the result of interference with functions that can be localized to specific regions of the laminin molecule.

\section{MATERIALS AND METHODS}

Isolation and culture of embryonic lungs. CD-1 strain (Charles River) mice were mated and the day a vaginal plug was found designated as Day 0. Pregnant females were sacrificed by cervical dislocation on Day 12 of gestation. Uteri were removed to sterile phosphate-buffered saline (PBS), and the lungs were dissected from the embryos with watchmakers forceps using a dissecting microscope. The lower right lobe was further dissected and cultured at the air-medium interface on the upper surface of polycarbonate filter membrane assemblies, $0.4-\mu \mathrm{m}$ pore size, (Millipore, Bedford, MA). The culture medium used was minimal essential medium, (GIBCO, Grand Island, NY) containing $10 \%$ fetal calf serum ( $\mathrm{Hy}-$ clone Lab, Logan, UT), nonessential amino acids (GIBCO), $0.29 \mathrm{mg} / \mathrm{ml} \mathrm{L-glutamine} \mathrm{(Irvine} \mathrm{Scientific,}$ Santa Ana, CA), $100 \mathrm{U} / \mathrm{ml}$ penicillin, $100 \mu \mathrm{g} / \mathrm{ml}$ streptomycin, and $0.25 \mu \mathrm{g} / \mathrm{ml}$ amphotericin B (Irvine Scientific). Cultures were incubated at $37^{\circ} \mathrm{C}$ in $5 \% \quad \mathrm{CO}_{2}$ and fed daily by a complete change of medium.

Lung explants maintained in organ culture continue to develop in vitro. The transparency of the tissue, plus the transparency of the polycarbonate filter in which the organ buds are grown, allows continuous monitoring of epithelial branching morphogenesis. By Day 12 of gestation (at the time that the lungs are dissected), the right lower lobe presents four to six terminal buds. After 3 days in culture there is a 10-fold or greater increase in the number of terminal buds.

Anti-laminin monoclonal antibodies. Five monoclonal antibodies (AL-1 to AL-5) were generated against laminin by immunization of male LOU/MNCr rats. The preparation, purification, and characterization of these antibodies have been previously described (Skubitz et al., 1987, 1988). All of the antibodies were of the IgG class except AL-5, which was an IgM; each antibody reacted with laminin by solid phase radioimmunoassay and radioimmunoprecipitation (Skubitz et al., 1987, 1988). The regions on laminin to which each antibody bound (Fig. 1) were determined by Western immunoblotting in combination with electron microscopy (Skubitz et al., 1987, 1988). Normal rat IgG and normal rat IgM were purchased from Cappel (Malvern, PA).

Conjugation of antibody with fluorochrome. For some studies, selected monoclonal antibodies were conjugated with fluorescein isothiocyanate (FITC) according to the technique described by Goding, (1976). Briefly, $2 \mathrm{mg} / \mathrm{ml}$ of antibody in $0.1 \mathrm{M}$ sodium carbonate buffer, $\mathrm{pH} 9.0$, was incubated with $50 \mu \mathrm{l}$ of FITC (Molecular Probes, Eugene, OR) dissolved in DMSO at $1 \mathrm{mg} / \mathrm{ml}$. This mixture was incubated at $4^{\circ} \mathrm{C}$ in the dark for $8 \mathrm{hr}$. Then $\mathrm{NH}_{4}$ $\mathrm{Cl}$ was added at a final concentration of $50 \mathrm{mM}$ and the mixture reaction was incubated at $4^{\circ} \mathrm{C}$ for another $2 \mathrm{hr}$. The reaction was stopped with xylene cyanol and glycerol at a final concentration of 0.1 and $5 \%$, respectively. The free FITC was separated from the FITC-antibody conjugates on a $25-\mathrm{ml} \mathrm{G}-50-80$ column (Sigma) equilibrated in $1 \times$ PBS. Optical densities per each antibody were taken (FITC at $495 \mathrm{~nm}$ and protein at $280 \mathrm{~nm}$ ) and the fluorochrome:protein ratio was calculated. All the ratios fell into the optimal range (0.3 to 1.0$)$.

Culture of lung explants in the presence of monoclonal antibodies to laminin and control immunoglobulins. Right lower lobes dissected from embryonic lungs at Day 12 of gestation were cultured for 3 days in the presence of various purified monoclonal antibodies or control normal rat immunoglobulins from the classes $G$ (IgG) and $\mathrm{M}(\operatorname{IgM})$. The antibodies, as well as the control IgG and IgM, were added to the wells in $100 \mu \mathrm{l}$ of PBS at a final concentration of 50 or $100 \mu \mathrm{g} / \mathrm{ml}$. The culture medium was replaced daily and antibody or control immunoglobulin replenished. For additional control experiments, the explants were left untreated. The experiments were repeated six times, providing a total of 12 explants for each antibody or control at $100 \mu \mathrm{g} / \mathrm{ml}$ and 8 explants for each antibody and control at $50 \mu \mathrm{g} / \mathrm{ml}$. In two additional experiments, anti-laminin antibodies at 
a concentration of $100 \mu \mathrm{g} / \mathrm{ml}$ were preadsorbed with 100 $\mu \mathrm{g}$ of laminin for $1 \mathrm{~h}$ prior to being introduced into the medium.

As previously reported (Schuger et al., 1990), branching morphogenesis was monitored and photographed using a phase-contrast microscope and the number of terminal branches was recorded daily. At the end of each experiment, the percentage of branching inhibition per explant was calculated as

No. terminal buds in treated explants (a)

$$
\begin{gathered}
\frac{- \text { No. terminal buds at time zero }(b)}{\text { No. terminal buds in control explants }(\mathrm{c})} \\
- \text { No. terminal buds at time zero }
\end{gathered}
$$

(a) Treated explants included those cultured in the presence of antibody or control immunoglobulin. (b) No. of terminal buds at time zero ranged from 4 to 6 . (c) Control explants were those left untreated.

Immunohistochemistry. Lungs removed from Day 12 embryos were immediately frozen in OCT embedding compound (Miles, Naperville, IL). Blocks were stored at $-70^{\circ} \mathrm{C}$ prior to sectioning. Ten-micrometer sections were cut and collected on polylysine-coated slides. Scctions were air dried and exposed in a moist chamber to $400 \mathrm{U} / \mathrm{ml}$ bovine testes hyaluronidase (Cappel) for 20 min. Sections were then exposed to $5 \%$ normal goat serum followed by the primary antibody for $2 \mathrm{hr}$ at room temperature. Monoclonal antibodies AL-1, AL-2, A-3, and AL-4 (all IgG's) and normal rat IgG were used at a dilution of 1:10; monoclonal antibody AL-5 (an IgM) and normal rat IgM were used at a dilution of 1:50. The sections were then washed in PBS and exposed to a 1:50 dilution of the secondary antibody (FITC-conjugated goat anti-rat IgG or IgM; Cappel) for $30 \mathrm{~min}$ at room temperature. Sections were washed extensively in PBS and coverslipped with glycerol containing $0.1 \%$ phenylenediamine (Sigma, St. Louis, MO). As an additional control, sections were also treated with just PBS containing no primary immunoglobulin. In further studies, tissue sections were consecutively exposed to two different monoclonal antibodies. For these experiments the second antibody was FITC-conjugated. The purpose of these studies was to determine possible sharing of tissue binding sites by different antibodies. If this is indeed the case, then it could be expected that incubation of a tissue section with the proper antibody will block further binding of a second, FITC-conjugated antibody. In contrast, if the two antibodies do not share common tissue binding sites then the positive stain observed with an FITC-conjugated antibody should not be abolished by previous exposure of the section to the first antibody.

To determine penetration and immunolocalization of the various monoclonal antibodies in the explants, tis- sues were incubated with the appropriate monoclonal antibody or with control immunoglobulin for $24 \mathrm{hr}$. Samples were then frozen in situ by filling the membrane assembly with OCT at $-20^{\circ} \mathrm{C}$. The samples were then processed for immunohistochemistry as above except that the primary antibody was omitted during the immunostaining procedure.

Light microscopy. Lung explants unexposed or exposed to the different antibodies were examined under light microscopy. For morphological detail and assessment of tissue preservalion after culcure, lung explants were fixed in $10 \%$ phosphate-buffered formalin, rinsed with PBS, and dehydrated through graded alcohols to $100 \%$. The samples were embedded in glycolmethacrylate (Polysciences, Warrington, PA). Five-micrometer sections were cut, mounted on slides, and stained with hematoxylin-eosin.

Mitotic activity was determined on frozen sections from lung explants exposed for $48 \mathrm{hr}$ to the various monoclonal antibodies $(100 \mu \mathrm{g} / \mathrm{ml})$. Control explants were left unexposed or exposed to IgG. Total number of mitotic figures in the mesenchymal and epithelial compartments was determined by sereening 16 fields (magnification 80) per explant.

\section{RESULTS}

Immunolocalization studies. All five of the monoclonal antibodies were immunoreactive with the embryonic lung tissue. AL-1, which in Western immunoblots recognizes the $A$ chain and binds to the cross region of laminin (Fig. 1), stained the epithelial basement membrane and, to a minor degree, the extracellular matrix compartment (Fig. 2A). AL-2, which recognizes A and B chains and binds to the long arm of the molecule near the cross region, presented a very similar pattern of reactivity (Fig. 2B). AL-3 and AL-4, which recognize $\mathrm{B}$ and A chains, respectively, and bind, the former to the inner rod segment of the B chain(s) and the latter to the carboxyl end of the A chain, stained only the epithelial basement membrane (Figs. 2C and 2D). Al-5, which binds to the globular segments of the lateral short arms (B chains), reacted weakly with the epithelial basement membrane and presented a pattern of diffuse staining throughout the extracellular matrix (Fig. 2E). None of the antibodies recognized vascular basement membranes. The data are summarized in Table 1.

Effects of monoclonal anti-laminin antibodies on lung branching morphogenesis. We next examined the effects of the monoclonal antibodies on lung morphogenesis. Branch formation was measured as described under Materials and Methods. Consistent with our previous report (Schuger et al., 1990), organ cultures of 12-day em- 

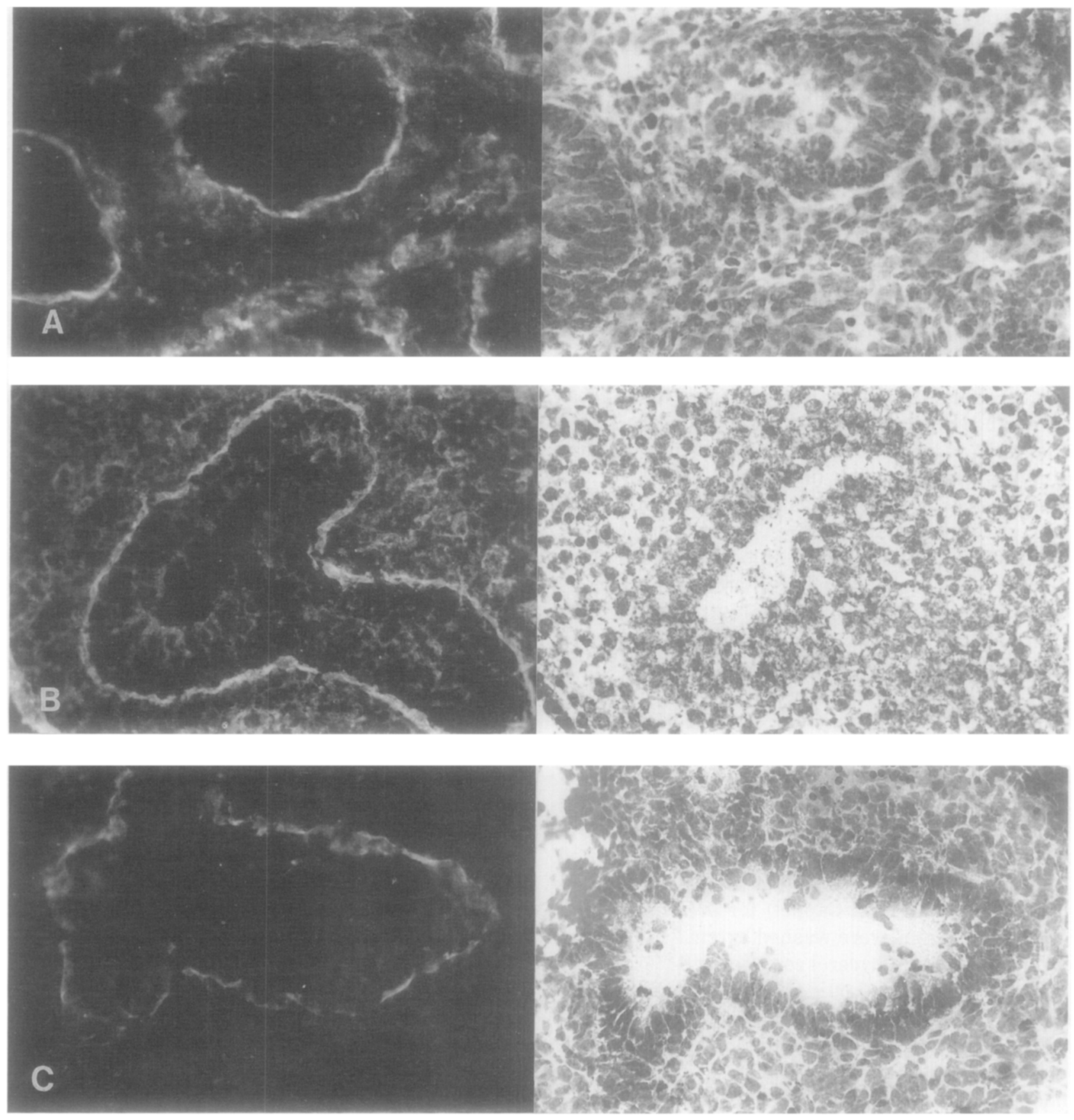

FIG. 2. Immunohistochemical localization of laminin in the 12-day embryonic mouse lung. The immunostain obtained with each antibody is shown in the corresponding H\&E counterstain for each is shown in the right-hand panels. An epithelial bud at Day 12 of gestation surrounded by mesenchyme is shown in each photograph. The airway lumen is approximately in the center of the photograph. Monoclonal antibodies AL-1 and AL-2 (A,B) stain predominantly the epithelial hasement membrane. The mesenchymal extracellular matrix is stained diffusely. Antibndies AL-3 and AL-4 (C,D) react exclusively with the epithelial basement membrane. Antibody AL-5 (E) appears to diffusely stain the extracellular matrix and the epithelial basement membrane. Normal rat immunoglobulin IgG does not stain any of the lung (F). Scale bar, $100 \mu \mathrm{m}$.

bryonic mouse lung underwent active branch formation over the subsequent 3 -day period. The mean number of terminal buds by the third day in control cultures was 53 $(S D=7$; range $49-80)$. Similar numbers of branches were observed in explants cultured in the presence of $100 \mu \mathrm{g} / \mathrm{ml}$ of normal rat IgG (Fig. $3 \mathrm{~A}$ ) or normal rat IgM or in the presence of an equal concentration of AL-2, AL-3 (Fig. 3C), or AL-4. In contrast, AL-1 and AL-5 

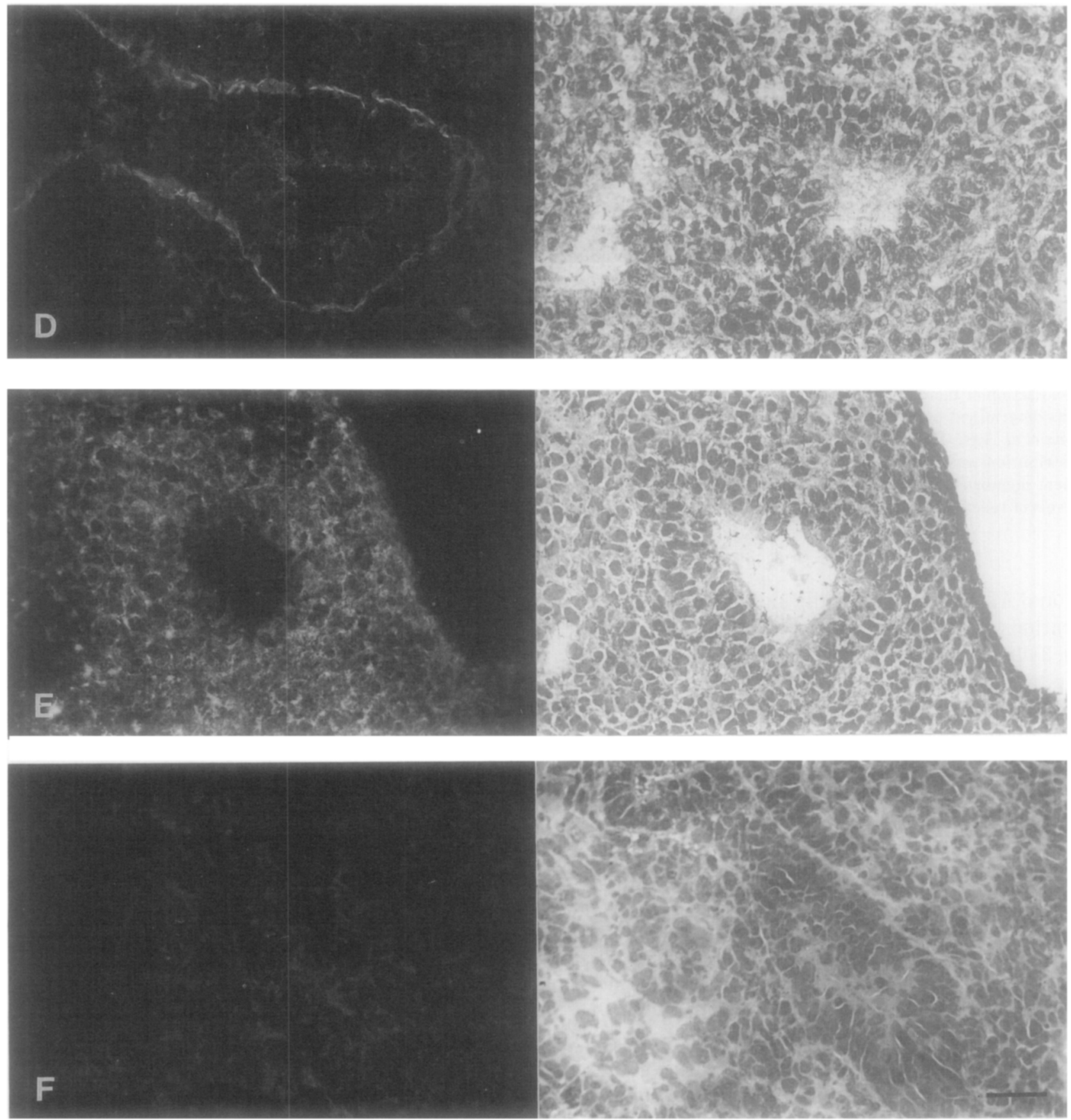

Fig. 2.-Continued

significantly decreased the number of terminal buds (Figs. 3E and 3G, respectively). Qualitative differences in the pattern of branching of the affected explants were observed with both antibodies. Their branching configuration was irregular and accompanied by segmental dilatation (Figs. 3E and 3G). Inhibition was concentration-dependent over the range $50-100 \mu \mathrm{g} / \mathrm{ml}$. The decrease in the number of branches was evident after 1 day in culture. By the second day in culture, at which time antibody effect was maximal, the percentage of branching inhibition was greater than $30 \%$ for antibody concentration of $100 \mu \mathrm{g} / \mathrm{ml}$ (Fig. 4) and $18 \%$ for antibody concentration of $50 \mu \mathrm{g} / \mathrm{ml}$ (not shown). Significant inhibition was still observed after 3 days but there was more variability among the different explants in the same treatment groups. The inhibition observed with 
TABLE 1

IMMUNOHISTOCHEMICAL STUDIES ON MOUSE EMBRYONIC LUNG (DAY 12 OF GESTATION)

\begin{tabular}{|c|c|c|c|}
\hline \multirow{2}{*}{$\begin{array}{c}\text { Monoclonal }^{\alpha} \\
\text { antibody }\end{array}$} & \multirow[b]{2}{*}{ Laminin domain $^{b}$} & \multicolumn{2}{|c|}{ Distribution $^{c}$} \\
\hline & & Diffuse & BM-Restricted \\
\hline AL-1 & Cross intersection & ++ & $+t+t$ \\
\hline AL-2 & Long arm below bifurcation & ++ & ++++ \\
\hline$\Lambda \mathrm{L}-3$ & Lateral arms, inner segment & 0 & ++++ \\
\hline AL-4 & Long arm, carboxy end & 0 & ++++ \\
\hline AL-5 & Lateral arms, outer segment & ++++ & ++ \\
\hline Normal IgG & - & 0 & 0 \\
\hline Normal IgM & - & 0 & 0 \\
\hline
\end{tabular}

${ }^{a}$ The generation and characterization of these antibodies have been previously described (Shubitz et al., 1987, 1988).

${ }^{b}$ Localization of monoclonal antibody binding to domains of laminin was determined by electron microscopy as previously described (Shubitz et al., 1987, 1988). See Fig. 1.

${ }^{c}$ Distribution was classified as either diffuse or restricted to the basement membrane (BM, No reactivity was scored as 0 , while positive levels of reactivity were scored from + (low) to ++++ (high).

AL-1 and AL-5 was statistically significant at both concentrations used ( 50 and $100 \mu \mathrm{g} / \mathrm{ml}$ ) after 1 and 2 days. After 3 days, statistical significance was observed only at the highest concentration. The effects of both AL-1 and AL-5 were abrogated by preadsorption with $100 \mu \mathrm{g}$ of laminin for $60 \mathrm{~min}$ prior to use.

Immunofluorescence localization of the antibodies in the lung explants after antibody treatment. Immunohistochemistry was used to assess the efficiency of the antibodies added to the organ cultures to penetrate the explants. For these experiments, lung explants were cultured for 2 days in the presence of monoclonal antibodies and control IgG or IgM as described under Materials and Methods. At the end of the treatment period, frozen sections obtained from the treated and control explants were exposed directly to an FITC-conjugated goat Fab fragment of anti-rat IgG or anti-rat IgM. These experiments showed that all of the monoclonal antibodies effectively penetrated the lung tissue during the course of the experiments and reacted with the appropriate tissue epitopes. The patterns of reactivity seen in the organ cultures were very similar to those observed originally when the antibodies were used for conventional immunohistochemistry on fresh, uncultured lungs. Antibodies AL-1, AL-2, AL-3, and AL-4 reacted primarily with the basement membrane while AL-5 was reactive with groups of cells in close proximity to the airway lumen (Figs. 3D, 3F, 3H). However, AL-1 and AL-2 immunoreactivity was not diffusely present in the extracellular matrix as was observed in the immunolocalization studies done on fresh, uncultured lungs.
At the end of the incubation period, lung explants were examined histologically for tissue viability. A complete preservation of tissue architecture was observed in all cases. Mitotic figures and occasional single necrotic cells were seen in both treated and control explants (Fig. 5). Thus, there were no cytological abnormalities associated with inhibition of branch formation.

Effects of monoclonal anti-laminin antibodies on mitotic activily. Lung explants were cultured for 2 days in the presence of monoclonal antibodies AL- 1 or AL-5 or in the presence of IgG as described under Materials and Methods. At the end of this period the number of mitotic figures was determined on frozen sections obtained from three explants exposed to AL-1, three explants exposed to AL-5, two exposed to $\operatorname{IgG}$, and one unexposed. Both AL-1 and AL-5 significantly decreased the number of mitotic figures in the epithelium compared to unexposed lungs or lungs exposed to control IgG. (In one experiment the epithelium of lung explants exposed to AL-1 and AL-5 presented 45 and 40 mitotic figures/explant, respectively, compared to 73 in IgG exposed explant.) However, we did not see a decrease in mitotic figures in the mesenchymal compartment of the AL-1and AL-5-treated explants. In fact, there was actually an increase in the number of mesenchymal mitotic figures in these explants. In the experiment presented above, the mesenchyme of lungs exposed to AL-1 and AL-5 showed 43 and 40 mitotic figures/explant, respectively, compared to 22 in IgG exposed explant. The experiments were repeated in three instances with similar results. The significance of this is not known at present.

Immunohistochemical studies with a combination of unlabeled and FITC-labeled monoclonal antibodies. Immunohistochemistry was used to determine whether the positive stain obtained with FITC-conjugated antibodies AL- 1 and AL-5 could be blocked by preincubation of the tissue sections with unconjugated AL-1 or AL-5. In these studies preincubation of the tissue sections with unconjugated AL-1 did not inhibit binding of AL-5FITC. AL-5-FITC presented a positive pattern identical to that obtained by conventional immunohistochemistry. As expected, unconjugated AL-5 inhibited binding of FITC-conjugated AL-5. Unfortunately, AL-1FITC did not bind to the tissue sections, even under control conditions.

\section{DISCUSSION}

In previous studies we found that when embryonic lung explants were cultured in the presence of polyclonal antibodies to laminin, branching morphogenesis was inhibited and gross abnormalities occurred (Schuger et al., 1990). Like other extracellular matrix 
glycoproteins, laminin is a multidomain macromolecule and its diverse biological activities are mediated by different segments of the protein in a way that seems to be cell-type or function specific. In order to determine whether the involvement of laminin in branching morphogenesis could be further localized to a particular domain, embryonic lung explants were cultured in the presence of monoclonal antibodies to various domains of the laminin molecule and their effect on morphogenesis was determined. The laminin domains to which each of the antibodies used in the present study localize have been established in previous reports by electron microscopy, and the laminin chains to which each antibody reacts have been determined by immunoblotting and radioimmunoassay (Skubitz el al., 1987, 1988). All of the domains recognized by the antibodies have been reported to promote cell attachment (Terranova el al., 1983; Graf et al., 1987; Aumailley et al., 1987; Skubitz et al., 1987; Charonis et al., 1988; Kouzi-Koliakos et al., 1989; Kleinman et al., 1989; Tashiro et al., 1989). In addition, some of these domains have heparin binding activity (Skubitz et al., 1988).

In the present study monoclonal antibodies were used first to determine laminin distribution in the 12th day embryonic lung and second to evaluate the functional involvement of different laminin domains in lung morphogenesis. The immunohistochemical studies localized laminin predominantly to the basement membrane. However, some immunoreactivity was also diffusely present in the extracellular matrix. Since it is unlikely that laminin produced by epithelial cells extends beyond the basement membrane into the mesenchymal extracellular matrix, our findings suggest that laminin is expressed by both the epithelial and the mesenchymal cells. These findings coincide with previous studies conducted on other developmental systems, in which a dual tissue origin of laminin was demonstrated (Simon-Assmann et al., 1988). The fact that not all of the monoclonal antibodies reacted with the laminin diffusely present in the extracellular matrix might indicate the existence of molecular isoforms differing structurally from each other. Alternatively, the lack of diffuse extracellular matrix staining with certain monoclonal antibodies may be due to masking of the appropriate epitopes.

Blocking experiments to determine the functional involvement of laminin in lung morphogenesis showed that two of the five antibodies interfered with organogenesis: antibody AL-1, which binds at the cross region of laminin, and antibody AL-5, which recognizes the globular segments of the B chain(s) (Skubitz et al., 1987, 1988). Both antibodies inhibited branching morphogenesis in a concentration-dependent manner and this effect was abrogated by preincubation with laminin. Lung explants exposed to AL-5 presented severe alterations in the pattern of branching. These kind of alterations were uncommon in those explants exposed to AL-1, suggesting that the two antibodies may be blocking different laminin functions. There was no evidence of cytotoxicity with either AL-1 or AL-5. However, explants exposed to both antibodies were smaller than controls and presented a decrease in the total number of mitotic figures. Interestingly, the inhibition in mitotic activity appeared to be specific for epithelial cells. There was no inhibition in the mesenchymal compartment. In fact, mitotic figures were prominent among mesenchymal cells. These observations suggest a complex effect of the antibodies on lung organogenesis rather than a general antiproliferative activity.

Failure of the other three monoclonal antibodies to interfere with branching morphogenesis was not due to inability to penetrate the lung tissue in organ culture, since immunolocalization studies demonstrated that during culture all five antibodies penetrated the lung and bound to the basement membrane or, in the case of AL-5, reacted with cells close to the basement membrane.

It should be noted that the binding sites of antibodies AL-2 and AL-3, which did not affect branching, present partial overlap with the binding site of AL-1, which inhibited branching activity. The difference in effect may indicate that among these antibodies, the effective one is that which is able to bind at high concentration to a relatively small key segment in the molecule. Even more, blocking of critical amino acid scquences might be required to obtain an effect. Studies using synthetic peptides representing sequences from critical laminin sites will help to answer this question.

The current studies indicate that specific determinants of laminin appear to be required in order for the laminin molecule to be functionally active during lung morphogenesis. In previous studies using polyclonal antibodies to laminin, we could not rule out the possibility that inhibition of morphogenesis was caused merely by steric hindrance of the polyclonal antibody bound to the basement membrane. However, in this study using monoclonal antibodies we can ensure that only specific domains of laminin are blocked by each of the antibodies. Rased on our results, we can suggest the following scenario to account for this inhibition of branching morphogenesis with monoclonal antibodies. Laminin synthesized by epithelial cells and probably also by mesenchymal cells becomes organized into a hasement membrane. Epithelial cell receptors for laminin recognize segments at the cross-shaped part of the molecule or in the globular region of the short arms. The cells bind to these parts of laminin and carry out the func- 

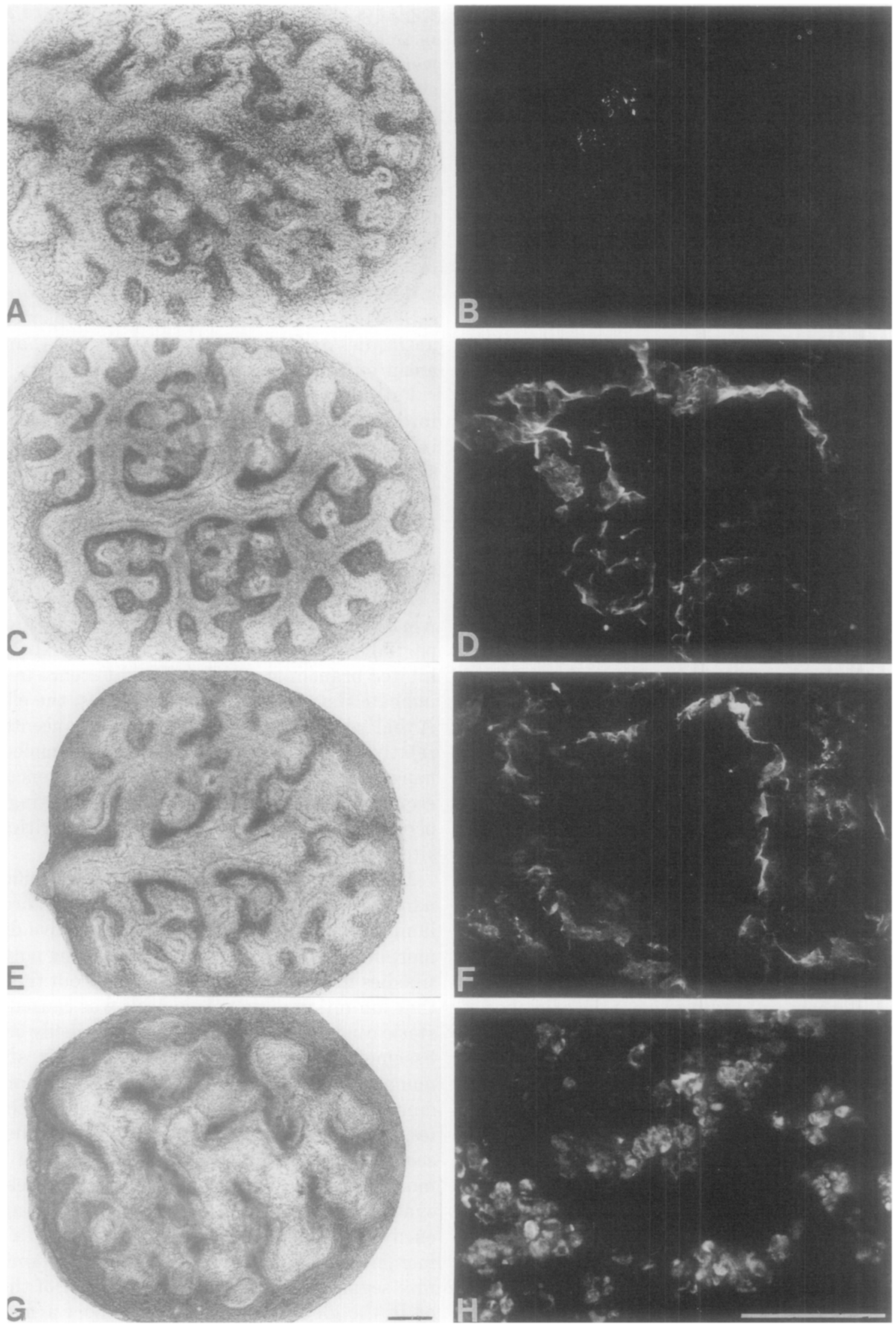
tions needed for normal morphogenesis. Alternatively, inhibition of normal morphogenesis might not be the result of blocking laminin functions in the basement membrane but rather of the blocking of laminin diffusely present in the extracellular matrix. In such case, it may be the mesenchymal cell interaction with laminin that is affected. While this is speculative, the finding that the two antibodies that inhibited morphogenesis showed diffuse extracellular matrix reactivity supports such a possibility.

With regard to AL-5 specifically, this antibody presented a staining pattern that was distinct from the other antibodies. This antibody stained the mesenchymal compartment but failed to stain the basement membrane. On this basis, it could be suggested that AL5 reactivity was due to interaction with molecules other than laminin however this is unlikely. Rotary shadow electron microscopy studies clearly demonstrated that AL-5 binds to a specific laminin region. This region, which is located at the terminal part of the lateral arms, has both cell binding and heparin binding activity (Skubitz, 1987, 1988; Kouzi-Koliakos, 1989). AL-5 inhibits both heparin binding to laminin and cell adhesion to laminin (Skubitz, 1987, 1988; Kouzi-Koliakos, 1989). It is evident from this that AL-5 recognizes epitopes on the laminin molecule and interferes with functions that are dependent on the same region of the molecule in which the epitopes reside. It should be pointed out that mesenchymal cells isolated from 12-day mouse embryo lungs synthesize laminin in primary culture (manuscript in preparation). Therefore, it would not be unreasonable to see staining of the mesenchymal compartment. Further while the lack of basement membrane staining with AL-5 is curious, it may be possible that the epitope for this antibody is either masked or not present in sufficient quantity to elicit a response with this antibody. (If the antibody were recognizing something in addition to laminin, this would not explain its lack of staining of the basement membrane.) Interestingly, it appears that the lack of basement membrane staining with AL-5 is developmentally regulated. When lungs form 14-day embryos are stained with this antibody, there is basement membrane staining as well as staining of the mesenchymal compartment (manuscript in preparation). Most importantly, incubation of soluble laminin with AL-5 completely abrogated its ability to interfere with normal mouse lung development. This is identical to what we saw when soluble laminin was incubated with $\mathrm{AL}-1$, the other antibody which interferes with lung development. If a moiety other than laminin were responsible for reactivity with this antibody, soluble laminin would not be expected to compete. Taken together, these data strongly suggest that laminin is the antigen for $A L-5$ and that the unusual staining pattern seen in the 12-day mouse embryo lungs cannot be explained on the basis of failure to react with laminin.

How specific domains of laminin act to promote branching morphogenesis and how antibodies interfere with their biological activity are important questions that remain to be elucidated. Many possibilities should be considered. In recent years it has become clear that cell attachment, proliferation, migration, and polarization (all essential to normal morphogenesis) result from cell interactions with specific laminin domains. It is, therefore, possible that the two laminin domains defined in this study mediate one or more of the above functions. It is even possible that each of these domains serves a different function during lung development and that blocking of morphogenesis is an end result of interfering with one of several diverse biological processes. The ability of several laminin domains to promote cell attachment in a cell-type-dependent manner has been extensively documented. Both the cross region of laminin and the globular domains of the B chain(s) have been reported to promote the attachment of a variety of cells. (Terranova et al., 1983; Graf et al., 1987; Charonis et al., 1988, Kouzi-Koliakos et al., 1989; Kleinman et al., 1989; Tashiro et al., 1989). Proliferation of epithelial and mesenchymal cells, the two main cell populations of the embryonic lung, is an anchorage-dependent phenomenon. If the two laminin domains defined in this study promote the attachment of embryonic lung cells, then cell proliferation and consequentially branching morphogenesis could be facilitated by these domains. It follows that interfering with the functions of these critical domains could lead to an inhibition of branching morphogenesis. Conversely, if the binding of laminin to other basement membrane constituents such as proteoglycans or even laminin itself (Timpl, 1989) is perturbed, then a proper assembly of the basement membrane may not take place. This could also result in impaired morphogenesis. It should be noted in this regard that the

FIG. 3. Left-hand column: Effects of monoclonal antibodies on branching morphogenesis. Lung organ cultures were established on Day 0 and incubated for 2 days in the presence of $100 \mu \mathrm{g} / \mathrm{ml}$ of normal rat IgG (A), antibody AL-3 (C), antibody AL-1 (E), or antibody AL-5 (G). Normal branching morphogenesis occurred in the explants exposed to IgG (A) or AL-3 (C). Inhibition in branching activity is evident in the cultures exposed to AL-1 (E) and AL-5 (G). Right-hand column: Assessment of antibody penetration into the explant during organ culture treatment. No immunoglobulin is bound to the tissue when the explants are cultured in the presence of normal rat IgG (B). When explants are cultured in the presence of AL-3 (D) or AL-1 (F), the antibodies penetrate the tissue and bind to the basement membrane. AL-5 reacts with clusters of cells close to the basement membrane (H). Scale bars, $100 \mu \mathrm{m}$. 


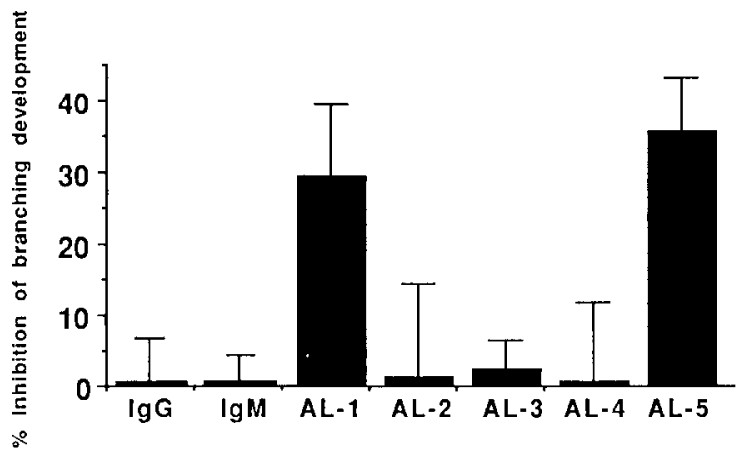

FIG. 4. Lung organ cultures were established on Day 0 and incubated for 2 days in the presence of $100 \mu \mathrm{g} / \mathrm{ml}$ of normal rat IgG, normal rat IgM, or mononuclear antibodies AL-1, Al-2, Al-3, AL-4, and AL-5. The percentage of branching inhibition was then calculated according to the formula shown under Materials and Methods. Percentages of inhibition \pm standard deviations are represented in this figure. Branching inhibition was observed in the explants exposed to AL-1 and AL-5. Both were statistically significant $(P>0.005, \mathrm{AL}-1 ; P>0.0001$, AL-5).

domain of laminin that recognized antibody AL-5 has heparin binding activity (Skubitz et al., 1988) and sequences of amino acids from this domain have been found to promote both cell adhesion and heparin binding (Charonis et al., 1988; Kouzi-Koliakos et al., 1989).

Not all of the domains with cell and/or heparin binding activity appear to be involved in branching morphogenesis. The YIGSR-containing sequence, located near the intersection of the cross on the B1 chain of laminin (i.e., the domain recognized by antibody AL-3), promotes the attachment of numerous cell lines (Graf et al., 1987; Iwamoto et al., 1988), whereas the globular end of the long arm, another established cell binding domain (Aumailley et al., 1987; Goodman et al., 1987), is recog- nized by AL-4. However, antibodies against these domains did not affect morphogenesis. It can be suggested that although laminin contains multiple cell adhesionpromoting domains, not all of them play a role in branching morphogenesis at this stage of lung development.

In addition to facilitating adhesion, laminin is also known to have direct mitogenic activity for some cells. This effect has been localized to the inner segments of the short arms, close to the cross intersection in regions that are rich in epidermal growth factor-like repeats in their amino acid sequence (Panayotou et al., 1989). The lack of inhibitory effect on branching development with antibody AL-3 (which binds to this region) seems to indicate that the effect of laminin on lung morphogenesis is not related to epidermal growth factor-like activity. Likewise, the long arm of the laminin molecule has been reported to play a role in the induction of polarization of tubular epithelium during embryonic kidney development (Klein et al., 1988). Antibody AL-2, directed against this region, did not alter epithelial polarization of the newly formed airways. The lack of effect with this antibody might indicate that cell polarization in the lung involves different mechanisms than those acting in the kidney. The process of conversion of mesenchyme into an epithelium, as occurs in the kidney, is quite different from the same process in the lung, in which a fully polarized epithelium proliferates and branches.

In conclusion, two regions of the laminin molecule (i.e., the cross section and the globular regions of the lateral short arms) play a role in lung branching morphogenesis. Both domains regulate epithelium and mesenchyme growth and branching morphogenesis. Both domains have in common their expression in the base-
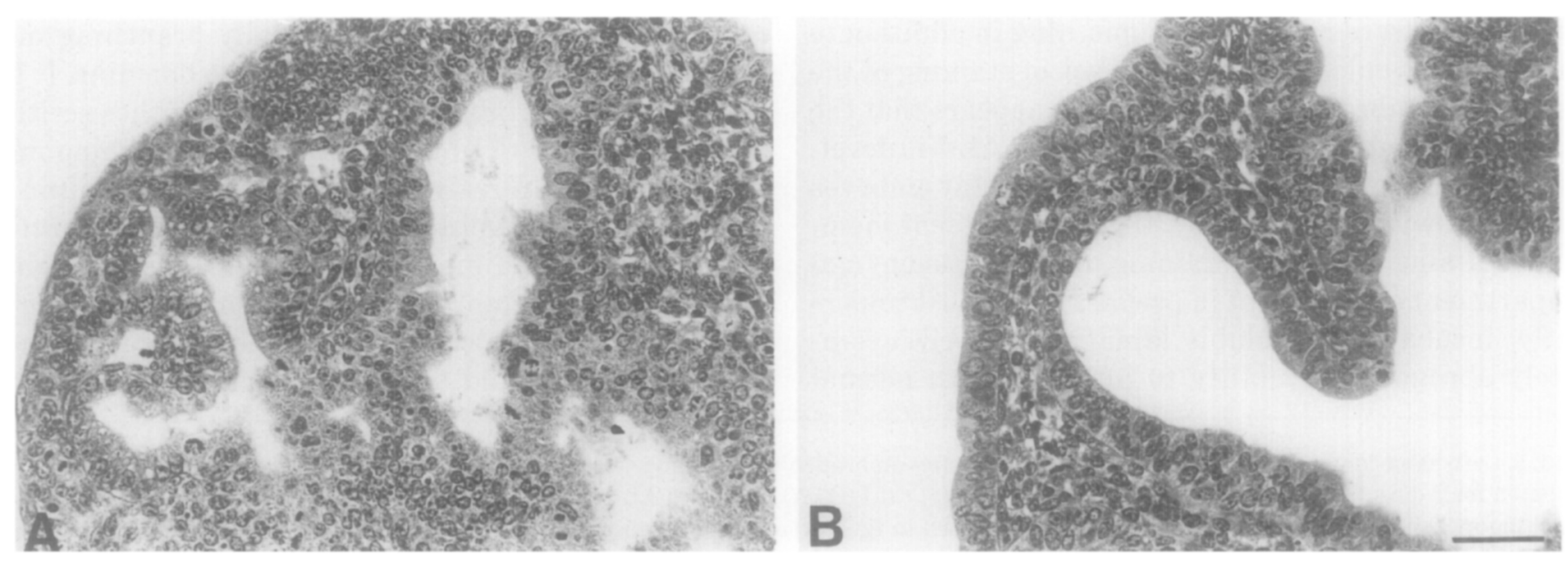

Fig. 5. Histological examination of mouse lung tissue in organ culturc. Longitudinal sections through explants maintained in culture for 3 days in the presence of $100 \mu \mathrm{g} / \mathrm{ml}$ of normal rat IgG (A) or in $100 \mu \mathrm{g} / \mathrm{ml}$ of AL-5 (B). The tissues demonstrate good viability with full architectural presentation. Seale bar, $50 \mu \mathrm{m}$. 
ment membrane as well as diffuse expression in the extracellular matrix.

This study was supported in part by a grant from the Tobacco Research Council (L.S.) and by Grant 1891179 from the Juvenile Diabetes Foundation (A.P.N.S.).

\section{REFERENCES}

Aumailley, M., Nurcombe, V., Edgar, D., Paulsson, M., and Timpl, R. (1987). The cellular interactions of laminin fragments. Cell adhesion correlates with two fragment-specific high affinity binding sites. J. Biol. Chem. 262, 11,532-11,538.

Charonis, A. S., Skubitz, A. P. N., Koliakos, G. G., Reger, L. A., DEgE, J., Vogel, A. M., WoHlhUETER, R., and FurChT, L. T. (1988). A novel synthetic peptide from the Bl chain of laminin with heparin-binding and cell adhesion-promoting activities. J. Cell Biol. 107, $1253-1260$.

CoOPer, A. R., and MacQueen, H. A. (1983). Subunits of laminin are differentially synthesized in mouse eggs and early embryos. Dev. Biol. 96, 467-471.

Ekblom, P., Alitalo, K., Vaheri, A., Timpl, R., and Saxen, L. (1980). Induction of a basement membrane glycoprotein in embryonic kidney: Possible role of laminin in morphogenesis. Proc. Natl. Acad. Sci. USA 77, 485-489.

Ekblom, P., Vestweber, D., and Kemler, R. (1986). Cell-matrix interactions and cell adhesion during development. Annu. Rev. Cell Biol. 2, $27-47$.

Ekblom, M., Klein, G., Mugraver, G., Fecker, L., Deutzmann, R., Timpl, R., and Ekblom, P. (1990). Transient and locally restricted expression of laminin A chain mRNA by developing epithelial cells during kidney organogenesis. Cell 60, 337-346.

GoDING, J. W. (1976). Conjugation of antibodies with fluorochromes: Modifications to the standard methods. J. Immunol. Methods 13, 215-226.

Goodman, S., Deutzmann, R., and von Der Mark, K. (1987). Two distinct cell-binding domains in laminin can independently promote non-neuronal cell adhesion and spreading. J. Cell Biol. 105, 589-598.

Graf, J., Iwamoto, Y., Sasaki, M., Martin, G. R., Kleinman, H. K., RobeY, F., and Yamada, Y. (1987). Identification of an amino acid sequence of laminin mediating cell attachment, chemotaxis, and receptor binding. Cell 48, 989-996.

Iwamoto, Y., Graf, J., Sasaki, M., Kleinman, H. K., Greatorex, D. R., Martin, G. R., RobeY, F. A., and Yamada, Y. (1988). Syntetic pentapeptide from $\mathrm{Bl}$ chain of laminin promotes B16F10 melanoma cell migration. J. Cell Physiol. 134, 287-291.

Klein, G., Langegger, M., Timpl, R., and Ekblom, P. (1988). Role of laminin A chain in the development of epithelial cell polarity. Cell 55, 331-341.

Kleinman, H., Ebihara, I., Killen, P., Sasaki, M., Cannon, F. B., YAMADA, Y., and MARTIN, G. R. (1987a). Genes for basement membrane proteins are coordinately expressed in differentiating F9 cells but not in normal adult murine tissues. Dev. Biol. 122, 373-378.

Kleinman, H. K., Graf, J., Iwamoto, Y., Kitten, R. C., Oegle, R. C., Sasaki, M., Yamada, Y., Martin, G. R., and Luckenbill-EDds, L. (1987b). Role of basement membranes in cell differentiation. Ann. NY Acad. Sci. 513, 134-145.

Kleinman, H. K., Graf, J., Iwamoto, Y., Sasaki, M., Schasteen, C. S., Yamada, Y., Martin, G. R., and Robey, F. A. (1989). Identifi- cation of a second active site in laminin for promotion of cell adhesion and migration and inhibition of in vivo melanoma lung colonization. Arch. Biochem. Biophys. 272, 39-45.

Kouzi-Koliakos, K., Koliakos, G. G., Tsilibary, E. C., Furcht, L. T., and Charonis, A. S. (1989). Mapping of three major heparin binding sites on laminin and identification of a novel heparin-binding site on the B1 chain. J. Biol. Chem. 264, 17.971-17,978.

Laurie, G. W., Horikoshi, S., Killen, P. D., Segui-Real, B., and YAMADA, Y. (1989). In situ hybridization reveals temporal and spatial changes in cellular expression of mRNA for a laminin receptor, laminin, and basement membrane (type IV) collagen in the developing kidney. J. Cell Biol. 109, 1352-1362.

MARTIN, G. R., and TIMPL, R. (1987). Laminin and other basement membrane components. In Annual Review of Cell Biology (G. E. Palade, B. M. Alberts, and J. A. Spunich, Eds.), Vol. 3., pp. $57-85$. Annual Reviews Inc., Palo Alto, CA.

Panayotou G., End, P., Aumailley, M., Timpl, R., and Engel, J. (1989). Domains of laminin with growth-factor activity. Cell $\mathbf{5 6}, 93-$ 101.

Sasaki, M., Kato, S., Kohno, K., Martin, G. R., and Yamada, Y. (1987). Sequence of the cDNA encoding the laminin B1 chain reveals a multidomain protein containing cysteine-rich repeats. Pro. Natl. Acad. Sci. USA 84, 935-939.

SASAKI, M., and YAMADA, Y. (1987). The laminin B2 chain has a multidomain structure homologous to the B1 chain. J. Biol. Chem. 262, 17,111-17,117.

Sasaki, M., Kleinman, H. K., Huber, H., Deutzmann, R., and YAMADA, Y. (1988). Laminin, a multidomain protein. The A chain has a unique domain and homology with the basement membrane proteglycan and the laminin B chains. J. Biol. Chem. 263, 16,536-16,544.

Schuger, L., O'Shea, S., Rheinheimer, J., and VARaNI, J. (1990). Laminin in lung development: Effects of anti laminin antibody in murine lung morphogenesis. Dev. Biol. 137, 26-32.

Simon-Assmann, P., Bouziges, F., Arnold, C., Haffen, K., and KEDINGER, M. (1988), Epithelial-mesenchymal interactions in the production of basement membrane components in the gut. Development 102, 339-347.

Skuritz, A. P. N., Charonis, A. S., Tsilibary, E. C., and FurCht, L. T. (1987). Localization of a tumor cell adhesion domain of laminin by a monoclonal antibody. Exp. Cell Res. 173, 349-360.

Skubitz, A. P. N., MCCARthy, J. B., Charonis, A. S., and FurCht, L. T. (1988). Localization of three distinct heparin-binding domains of laminin by monoclonal antibodies. J. Biol. Chem. 263, 4,861-4,868.

Tashiro, K., Sephel, G. C., Weeks, B., Sasaki, M., Martin, G. R., Kleinman, H. K., and Yamada, Y. (1989). A synthetic peptide containing the IKVAV sequence from the A chain of laminin mediates cell attachment, migration and neuritic outgrowth. $J$. Biol. Chem. 264, 16,174-16,182.

Thrranova, V. P., RaO, C. N., Katebic, T., Margitume, I. M., and LioTTA, L. A. (1983). Laminin receptor on human breast carcinoma cells. Proc. Natl. Acad. Sci. USA 80, 444-448.

Timpl, R., RHODE, M., GeHron-Robey, P., Rennard, S. T., Foidart, J. M., and MARTIN, G. R. (1979). Laminin-A glycoprotein from basement membranes. .J. Biol. Chem. 254, 9933-9937.

TIMPL, R. (1989). Structure and biological activity of basement membrane proteins. Eur. J. Biochem. 180, 487-502.

Wu, T. C., WaN, Y. J., Chung, A. E., and Damajonov, I. (1983). Immunohistochemical localization of entactin and laminin in mouse embryos and fetuses. Den. Biol. 100, 496-505. 